\title{
A Equipe na Estratégia de Saúde da Família: uma Experiência do PET-Saúde
}

\author{
The Family Health Strategy Team: an \\ Experience with the Educational Program for \\ Health Work
}

\author{
Thiago Nogueira Silva \\ Natália Maria Tavares Ferreira Borges ${ }^{1}$ \\ Melissa Mota Santana \\ Mayara Novais Pereira \\ Paula Nascimento Pignaton ${ }^{I}$ \\ Janaina Santos Sacramento \\ Maria de Fátima Aquino ${ }^{I I}$ \\ Márcia Verônica Caldeira Quadros ${ }^{I}$ \\ Regina Lino Vieira \\ Adélia Maria Carvalho de Melo
}

\footnotetext{
PALAVRAS-CHAVE:

- Saúde da Família;

- Pessoal de Saúde;

- Formação de Recursos

Humanos;

- Relações ComunidadeInstituição.
}

Recebido em: 20/09/2010

Aprovado em: 19/11/2010

\section{RESUMO}

O trabalho em saúde realizado em termos da Atenção Básica por meio da Estratégia Saúde da Família (ESF) tem-se caracterizado por uma divisão parcelar do trabalho entre profissionais de saúde cada vez mais especializados e dependentes do trabalho de outros, perdendo, assim, autonomia e resolutividade. Percebe-se a atuação de equipes extremamente fragmentadas, nas quais cada profissional meramente executa a parte que lhe cabe no processo, sem se preocupar com o resultado geral e abstendo-se do seu papel social. O objetivo deste trabalho é relatar como essa tendência pôde ser verificada em uma Equipe de Saúde da Família no bairro do Salobrinho, no município de Ilhéus - BA, por universitários participantes do Programa de Ensino pelo Trabalho para a Saúde (PET-Saúde) vinculado à Universidade Estadual de Santa Cruz, Ilhéus - BA, e sua tentativa de modificar a realidade a partir do uso de metodologias participativas para valorização da equipe.

\begin{abstract}
Work in primary healthcare under the Family Health Strategy has shown a division of labor between increasingly specialized health professionals that are dependent on the work of others, thus losing autonomy and case-resolution capacity. Such health teams display extremely fragmented work, in which individual professionals merely carry out their part in the process, without worrying about the overall results and abstaining from their social role. The aim of this article was to report on this trend in a Family Health team in the neighborhood of Salobrinho, Ilhéus, Bahia State, Brazil, through university students participating in the Educational Program for Health Work (PET-Saúde), affiliated with the Santa Cruz State University, Ilhéus, Bahia, and their attempt to change the reality using participatory methodologies for valuing teamwork.
\end{abstract}




\section{INTRODUÇÃO}

A formulação da Constituição de 1988, que ampliou os direitos do cidadão e apresentou a soberania popular como um de seus princípios, afirmou pela primeira vez no País a saúde como um direito de todos e um dever do Estado1. O advento da Reforma Sanitária, com a criação do Sistema Único de Saúde (SUS), trouxe para o Brasil novas perspectivas no campo do cuidado. A partir daí, surgem políticas públicas de saúde voltadas para assegurar esse direito ao cidadão brasileiro, representadas principalmente pela Lei 8.080/90, que dispõe sobre as condições para a promoção, proteção e recuperação da saúde, a organização e o funcionamento dos serviços correspondentes e dá outras providências. As políticas e diretrizes desse novo sistema surgiram da necessidade de mudança do modelo assistencial à saúde no País, migrando do modelo médico-assistencialista para um modelo de promoção à saúde como partícipe no processo de transformação de sua própria realidade, centrado nas necessidades do indivíduo e observando-se suas várias dimensões.

Nesse contexto, o Programa Saúde da Família (PSF) configura-se a principal estratégia do SUS para a reorientação das práticas em saúde. Tal modelo aposta na equipe como instrumento de operacionalização dos princípios da atenção básica, tendo como elementos centrais: o trabalho com adscrição da clientela; a apropriação do território; o acolhimento como porta de entrada para as Unidades de Saúde da Família (USF); a visita domiciliar; a integralidade das práticas; e a equipe multiprofissional. Embora o trabalho esteja direcionado para práticas multiprofissionais, nada garante, nas estratégias do PSF, que haverá ruptura com a dinâmica médico-centrada do modelo hegemônico atual. ${ }^{2}$ Nessa perspectiva de um modelo de saúde com caráter substitutivo, é necessário que os trabalhadores em saúde também estejam comprometidos com os desafios dessa mudança, observando-se a forma como essas práticas são produzidas e traduzidas em ações de saúde.

Desde o nascimento do SUS, a formação de profissionais em consonância com seus princípios tem-se constituído um desafio. Gil (2005) afirma que tem sido constatado que o perfil dos profissionais formados não é adequado o suficiente para prepará-los para uma atuação na perspectiva da atenção integral à saúde e de práticas que contemplem ações de promoção, proteção, prevenção, atenção precoce, cura e reabilitação ${ }^{3}$. A autora continua: "Em tese, todos os que atuam nesse âmbito da atenção (da saúde da família) deveriam estar aptos para compreender e agir sobre determinantes do processo saúde/ doença, o que implicaria realizar uma síntese de saberes a serem utilizados na definição dos cuidados dos indivíduos e famílias" ${ }^{\prime 3}$ (p.491). Ainda sobre esse tema, Merhy coloca que um dos "grandes nós críticos" das propostas que apostam na mudança do modelo tecnoassistencial em saúde no Brasil tem sido o modo como se estruturam e se gerenciam os processos de trabalho. ${ }^{4}$ (p.71).

Paralelamente, autores têm proposto caminhos para reorientar as práticas profissionais a partir da articulação entre ensino, gestão, atenção e controle social, e da análise crítica da educação que se tem feito no setor saúde ${ }^{5-6}$. Agrega-se a essa proposta o ideal do trabalho humano como absolutamente fundamental e insubstituível em saúde e o de que as pessoas são os recursos mais importantes para se produzir (ou não) mudanças no interior de uma organização. Outro aspecto fundamental na observação do processo de trabalho é o reconhecimento de que o trabalhador possui um razoável autogoverno no seu fazer cotidiano, ou seja, ele comanda como se dará a assistência. Faz-se impostergável, portanto, o desenvolvimento de um processo de formação e capacitação permanente de todos os profissionais envolvidos, para desenvolver-se a Estratégia Saúde da Família (ESF), sendo necessários outros saberes e novos instrumentos. Produzir saúde considerando as famílias em suas condições concretas de vida requer que tomemos saberes como os da cultura popular e da educação, produzidos por meio do diálogo, saberes que aportam a escuta, o vínculo, o acolhimento, a autonomização, entre outros ${ }^{2,7}$.

No âmbito da formação em nível superior de profissionais de saúde, a integração ensino-serviço promove a inserção de estudantes universitários na realidade dos serviços de saúde, propiciando um espaço de troca de experiências e conhecimento com ressignificação de saberes, enquanto sujeitos capazes de atuar na perspectiva da educação permanente ao mesmo tempo em que assumem uma formação em consonância com os princípios do SUS.

O artigo 200 da Constituição Federal de 1988 estabelece a competência dos órgãos gestores do SUS, para ordenar a formação dos recursos humanos em saúde e incrementar o desenvolvimento científico e tecnológico. Desde então surgiram estratégias para operacionalizar o que está previsto na Carta Magna ${ }^{1}$.

Em 2001, foram formuladas as Diretrizes Curriculares Nacionais dos cursos de graduação em Medicina, Enfermagem e Nutrição, que tratam de orientações para elaboração dos currículos pelas instituições de ensino superior, de tal forma a flexibilizar a formação oferecida aos estudantes, adequando-a ao sistema de saúde vigente no País ${ }^{8}$.

Com base nesse cenário sociopolítico, o Ministério da Saúde (MS), juntamente com o Ministério da Educação e Cultura (MEC), vem estimulando a parceria entre instituições formadoras de profissionais de saúde e serviços de saúde, para o 
desenvolvimento de projetos de ensino nessa área. Tal iniciativa busca viabilizar o desenvolvimento da formação de profissionais de saúde em consonância com os princípios do SUS e mais comprometido com as necessidades de saúde da população brasileira.

Como exemplo do estímulo a essas parcerias pode-se citar o Promed (Programa de Incentivo às Mudanças Curriculares nos Cursos de Medicina - Ministério da Saúde, 2002); o Versus (Vivências e Estágios na Realidade do Sistema Único de Saúde do Brasil - Ministério da Saúde, 2004), e o Pró-Saúde (Programa Nacional de Reorientação da Formação Profissional em Saúde - Ministério da Saúde, 2005). No campo da transdisciplinaridade, o Projeto Rondon também tem destaque como uma iniciativa do Ministério da Defesa que promove a vivência de universitários de diversas áreas em regiões com realidades distintas das suas. A aposta em iniciativas como essas se fundamenta na ideia de que a construção de um processo de ensino-aprendizagem crítico e reflexivo propicia ao futuro profissional de saúde a significação crítica da ordem social em que está inserida sua prática profissional. Mais do que capacitar tecnicamente, o investimento na organização de processos de ensino-aprendizagem tem a potência de abrir caminhos para que o aluno/profissional de saúde se torne agente das mudanças que a sociedade brasileira necessita para tornar o País mais equânime e justo.9 Além disso, esse processo de ensino tem a vantagem de tornar possível o aprendizado mais significativo através da articulação entre teoria e prática, na perspectiva da interdisciplinaridade.

Um das estratégias mais recentes de inserção de estudantes em serviços de saúde é o Programa de Educação pelo Trabalho para a Saúde - PET-Saúde, que foi instituído em 3 de setembro de 2008 por meio da portaria interministerial $n^{\underline{0}}$ 180210. O PET-Saúde visa a estimular o processo de integração ensino, serviço e capacitação pedagógica dos profissionais e estudantes na área de Atenção Básica (AB), com o intuito de beneficiar a comunidade de modo geral especialmente na estratégia de Saúde da Família. Os alunos participantes selecionados estão envolvidos com a iniciação ao trabalho e desenvolvimento de pesquisa na área de Atenção Básica. O PET-Saúde, portanto, surge como importante alternativa extensionista para a produção de conhecimento na prática em saúde, em especial, por meio da produção de cuidados desse campo de atenção e acerca das dimensões que envolvem os problemas encontrados na ESF. Põe em curso a experimentação de propostas com novos modos de produzir atos de saúde para intervir nos processos de trabalho, nas mudanças de práticas clínicas e sanitárias que promovem a incorporação da clínica ampliada interdisciplinar e multiprofissional, promovendo a autonomização dos sujeitos, o comprometimento e a responsabilização com a saúde individual e coletiva.

O objetivo do presente trabalho é relatar a experiência vivida por discentes universitários no contexto de uma equipe de Saúde da Família do sul da Bahia como uma das equipes integrantes do PET-Saúde vinculadas à Universidade Estadual de Santa Cruz (Uesc), em Ilhéus - BA.

\section{TRAJETÓRIA METODOLÓGICA}

Trata-se de um relato da experiência nas atividades desenvolvidas, entre março de 2009 e março de 2010, por alunos do curso de Medicina e Enfermagem, dois preceptores e uma tutora acadêmica da Uesc, integrantes de uma das equipes do PET-Saúde Ilhéus, também da Uesc.

Conforme proposta do PET-Saúde, os discentes de Enfermagem e Medicina passaram a acompanhar as atividades do PSF Salobrinho I, com carga horária semanal de $8 \mathrm{~h}$. A partir da observação participante e da descrição das atividades em diário de campo, foi possível levantar problemas no cerne da equipe, sendo esses posteriormente discutidos em reuniões semanais com a tutora acadêmica e em reuniões mensais com a outras seis equipes do PET-Saúde, as quais tinham vivência em outras unidades de saúde da família do município de Ilhéus. As ações que os estudantes desempenharam incluíram ainda análise dos dados Sistema de Informação da Atenção Básica (SIAB), bem como análise das fichas A, preenchidas pelos Agentes Comunitários de Saúde (ACS), para tentar estabelecer o perfil epidemiológico da população adscrita pelo PSF em questão. Todo esse processo tinha como objetivo final a priorização de uma linha de cuidado, ou seja, um modelo de atenção à saúde no qual "o usuário é o elemento estruturante de todo processo de produção da saúde, quebrando com um tradicional modo de intervir sobre o campo das necessidades, de forma compartimentada. Nesse caso, o trabalho é integrado e não partilhado, reunindo na cadeia produtiva do cuidado um saber fazer cada vez mais múltiplo"11.

Um primeiro encontro foi realizado para apresentar o projeto do PET-Saúde à equipe e, após quatro meses de ambientação e estabelecimento de vínculos, os estudantes promoveram quatro oficinas abordando os temas: as concepções de equipe, saúde e ESF; atribuições da ESF; territorialização; acolhimento e humanização. Foram utilizadas metodologias ativas de ensino-aprendizagem, buscando conhecer a percepção dos atores sociais sobre as temáticas e promover a reflexão sobre o processo de trabalho na ESF. O contexto de formulação dessas oficinas, sua caracterização, bem como os resultados encontrados são descritos a seguir. 


\section{RESULTADOS E DISCUSSÃO}

No início, foram encontradas dificuldades relacionadas à desestruturação e desmotivação da Equipe de Saúde da Família em relação ao seu trabalho, às quais foram demonstradas, por exemplo, pela resistência dos Agentes Comunitários de Saúde em apoiar a equipe do PET-Saúde e permitir que ela participasse da rotina de suas atividades. Percebeu-se que a equipe enfrentava um processo de assimetria de suas ações, evidenciado pelo distanciamento entre as práticas dos princípios e diretrizes da Atenção Básica. Aos poucos, compreendeu-se que tal comportamento era reflexo de uma intensa frustração dos profissionais com as práticas do serviço e com sua própria atuação na comunidade. A precarização das condições de trabalho, a alta rotatividade de médicos e enfermeiras, a inexistência de reuniões de equipe e a deficiência na oferta de atividades de educação permanente desencadearam um processo de perda de identidade da equipe com consequente fragilidade e desestímulo.

Diante desse contexto, o grupo PET-Saúde Salobrinho I priorizou a equipe como alvo/instrumento de produção de cuidados em saúde. Foram utilizadas estratégias de valorização dos sujeitos como peças fundamentais na construção efetiva do trabalho em saúde, por meio do estímulo à reflexão sobre o funcionamento da Atenção Básica, sobre o conceito de saúde e de equipe, e sobre a importância da aplicação dessa reflexão na prática do acolhimento e no fortalecimento do seu papel como profissional de saúde e como cidadão. Tal abordagem foi feita através de reuniões nas quais se discutia cada assunto de maneira diferente, utilizando recursos visuais, como apresentações de slides e realizações de atividades dinâmicas e participativas e, ainda, a construção de cartazes representativos de imagens, palavras ou frases-relato. Em cada reunião, utilizou-se também a estratégia das dinâmicas de sensibilização, que culminavam em uma reflexão acerca da importância de cada um e da necessidade da união e do empenho individual de todos para o alcance do bem coletivo.

A partir desses encontros, foi possível conhecer anseios e demandas da equipe, bem como suas trajetórias e histórias. Pôde-se analisar, conjuntamente, como vem-se estabelecendo o cuidado em saúde no espaço de trabalho, observando-se os princípios da Atenção Básica. Dessa forma, os atores identificaram dificuldades, potencialidades e propostas para avançar na implementação desses princípios. As metodologias utilizadas favoreceram o resgate da autoestima desses sujeitos, com valorização de seus saberes, por meio do qual a equipe se percebeu como recurso fundamental às práticas em saúde. A vivência na unidade promoveu espa- ços de escuta, partindo do pressuposto de que a comunicação é um recurso valioso no processo de ajuda, e a escuta é uma habilidade de auto e heterocompreensão. ${ }^{12}$ A ênfase no trabalho em equipe está pautada na ideia de que ele tem como objetivo a obtenção de impactos sobre os diferentes fatores que interferem no processo saúde-doença. A ação interdisciplinar pressupõe a possibilidade da prática de um profissional se reconstruir na prática do outro, ambos sendo transformados para a intervenção na realidade em que estão inseridos. Tais movimentos têm a força de impulsionar os sujeitos na construção de novos pactos de convivência e práticas que aproximem os serviços de saúde dos conceitos da atenção integral, humanizada e de qualidade, da equidade e dos demais marcos dos processos de reforma do sistema brasileiro de saúde ${ }^{13}$

A partir das experiências obtidas por meio da participação dos discentes, preceptores e tutora do PET-Saúde, decidiu-se realizar um projeto de pesquisa que tem como objetivo principal a construção de uma linha de cuidado relacionada à gestão de pessoas. Tal projeto serviria para tentar reafirmar a Estratégia Saúde da Família como principal estratégia de reorientação do modelo técnico-assistencial em saúde, ao passo que o fortalecimento da equipe é etapa fundamental para seu desenvolvimento.

O projeto ainda está em andamento, e o método a ser utilizado nesta pesquisa é o de pesquisa-ação, que busca descrever e interpretar a dinâmica cultural e social de um grupo em um determinado espaço geográfico.

As etapas predefinidas na pesquisa-ação consistem em: (1) Diagnóstico do problema, o qual vem sendo feito desde 2009 por meio das observações realizadas durante o acompanhamento rotineiro da dinâmica da unidade, das reuniões com a equipe e a partir de depoimentos dos membros da própria equipe; (2) Discussão e planejamento da ação; (3) Construção de questionário a fim de identificar a percepção sobre serviço de saúde, processo de trabalho, PSF, linhas de cuidado e análise da equipe após coleta dos dados; (4) Realização de oficinas sobre as dificuldades encontradas nos relatos e aplicação do mesmo questionário depois delas realizadas; (5) Execução da ação, a partir da qual se realiza a aplicação de questionários, análise das respostas dadas a eles e realização de oficinas oferecidas aos membros da equipe, objetivando a promoção da saúde da população - os assuntos das oficinas serão: equipe, saúde da mulher, crescimento, desenvolvimento e hiperdia; (6) Avaliação dos resultados, sendo realizada por meio de atividades como: resumos de reuniões administrativas ou de aprendizagem, anotações feitas pelos pesquisadores, relatórios de oficinas e diários de campo. Os 
resultados e produtos esperados serão avaliados pela análise das discussões grupais com a ESF, com o propósito de fazer uma aproximação com as realidades e necessidades percebidas; descrição dos benefícios de ações de educação permanente na qualificação do cuidado ofertado a grupos populacionais específicos; ressignificação dos processos de trabalho; e divulgação científica, com produção de artigos, envolvendo estudantes, docentes e profissionais de saúde da família, participantes do PET-Saúde.

\section{CONSIDERAÇÕES FINAIS}

Ainda que esta abordagem tenha contribuído bastante para a melhoria da situação de instabilidade da equipe, existem muitos aspectos a serem refletidos e repensados acerca da ESF, conforme preconizado pelo Ministério da Saúde. Em primeiro lugar, é preciso vencer a tendência à organização da produção da saúde com base em lógicas advindas da indústria, como a lógica taylorista, visto que o serviço de saúde não pode ser comparado a uma linha de produção, com a confecção de um simples produto material comercializável14. Em segundo lugar, é imprescindível uma gestão política comprometida com a produção adequada de cuidados em saúde e desarticulada dos interesses individuais, pessoais. Finalmente, faz-se necessária uma transformação na mentalidade dos profissionais de saúde, o que abrange não só mudanças na formação curricular desses profissionais (para as quais o PET-Saúde contribui de forma decisiva), como também profundas reflexões acerca do processo de trabalho em saúde que cada indivíduo opera na micropolítica, espaço onde atuam as diversas subjetividades. É nesse espaço de constante negociação que a equipe tem o potencial de agir em prol da coletividade, para que esta ganhe um real significado e vire uma bandeira de luta para os atores sociais envolvidos na produção do cuidado em saúde.

\section{REFERÊNCIAS}

1. Brasil. Constituição (1988). Constituição da República Federativa do Brasil. Brasília, DF: Senado; 1988.

2. Franco TB, Merhy EE. Programa de Saúde da Família (PSF): contradições de um programa destinado à mudança do modelo técnico-assistencial. In: Merhy EE. O Trabalho em Saúde: olhando e experimentando o SUS no cotidiano. São Paulo: Hucitec, 2003. [Acesso em 10 set. 2010] Disponível em: http: //www.uff.br/saudecoletiva/professores/ merhy/capitulos-14.pdf.

3. Gil CRR. Formação de Recursos Humanos em Saúde da Família: paradoxos e perspectivas. Cad de Saúde Pública 2005; 21 (2): 490-498.
4. Merhy EE. Em Busca do Tempo Perdido: a micropolítica do trabalho vivo em saúde. In: Merhy EE, Onocko R, org. Praxis en salud: un desafio para lo público. São Paulo: Hucitec; 1997. p. 71-112.

5. Ceccim RB. Educação Permanente em Saúde: descentralização e disseminação de capacidade pedagógica na saúde. Ver Ciência \& Saúde Coletiva 2005;10 (4): 975-986.

6. Ceccim RB, Feuerwerker LCM. O Quadrilátero da Formação para a Área da Saúde: ensino, gestão, atenção e contrle social. Rev. Saúde Coletiva 2004; 14(1): 41-65.

7. Araújo MBS, Rocha PM. Trabalho em Equipe: um desafio para a consolidação da estratégia saúde da família. Ciência \& Saúde Coletiva 2007; 12(2): 455-164.

8. Brasil. Ministério da Educação. Parecer CNE/CES n. 12001, aprovado em 7 de agosto de 2001. Diretrizes Curriculares Nacionais dos Cursos de Graduação em Enfermagem, Medicina e Nutrição [legislação na Internet]. Brasília; 2001. [Capturado em 18 set. 2010]. Disponível em: http: / /www.mec.gov.br/cne/default.shtm\#diret.

9. Pereira JG, Fracolli LA. A Contribuição da Articulação Ensino-Serviço para a Construção da Vigilância da Saúde: a perspectiva dos docentes. Rev Latino-Am Enferm. 2009; 17(2).

10. Brasil. Portaria Interministerial no. 1.802, de 26 de agosto de 2008 (BR). Institui o Programa de Educação pelo Trabalho para a Saúde - PET-Saúde. Diário Oficial da União, Poder Executivo, Brasília, 26 ago., 2008. Seção 1: 1677-7042.

11. Franco TB, Magalhães Júnior HM. Integralidade na Assistência a Saúde: a organização das linhas do cuidado. In: O Trabalho em Saúde: olhando e experienciando o SUS no cotidiano. 2 ed. São Paulo: HUCITEC; 2004.

12. Souza RC, Pereira MA; Kantorski LP. Escuta Terapêutica: instrumento essencial do cuidado em enfermagem. Rev Enferm UERJ 2003; 11: 92-97.

13. Ceccim RB. Educação Permanente em Saúde: desafio ambicioso e necessário. Interface - Comunic, Saúde, Educ 2004/2005; 9(16):161-77.

14. Ribeiro EM, Pires D, Blan VLG. A teorização sobre processo de trabalho em saúde como instrumental para análise do trabalho no Programa Saúde da Família. Cad. Saúde Pública 2004; 20(2): 438-446.

\section{CONTRIBUIÇÃO DOS AUTORES}

Thiago Nogueira Silva, Mayara Novais Pereira, Paula Nascimento Pignaton, Márcia Verônica Caldeira Quadros e Adélia Maria Carvalho de Melo participaram na redação do texto e revisão do artigo. Natália Maria Tavares Ferreira Borges participou na coleta de dados, redação do texto e revisão. Melissa 
Mota Santana, Janaína Santos Sacramento participou na redação do texto, levantamento bibliográfico do artigo. Maria de Fátima Aquino participou da redação do artigo. Regina Lino Vieira participou da produção do texto e revisão. Adélia Maria Carvalho de Melo

\section{CONFLITO DE INTERESSES}

Declarou não haver.

\section{ENDEREÇO PARA CORRESPONDÊNCIA}

Adélia Maria Carvalho de Melo

Universidade Estadual de Santa Cruz

Campus Soane Nazaré de Andrade, km 16

Rodovia Ilhéus - Itabuna - Ilhéus

CEP. 45662-000 BA

E-mail: adelia@uesc.br 